\title{
An Investigation of Two Acoustic Propagation Codes for Three-Dimensional Geometries
}

\author{
D. M. Nark, W. R. Watson; and M. G. Jones \\ NASA Langley Research Center
}

\begin{abstract}
The ability to predict fan noise within complex three-dimensional aircraft engine nacelle geometries is a valuable tool in studying low-noise designs. Recent years have seen the development of aeroacoustic propagation codes using various levels of approximation to obtain such a capability. In light of this, it is beneficial to pursue a design paradigm that incorporates the strengths of the various tools. The development of a quasi-3D methodology (Q3D-FEM) at NASA Langley has brought these ideas to mind in relation to the framework of the CDUCT-LaRC acoustic propagation and radiation tool. As more extensive three dimensional codes become available, it would seem appropriate to incorporate these tools into a framework similar to CDUCT-LaRC and use them in a complementary manner. This work focuses on such an approach in beginning the steps toward a systematic assessment of the errors, and hence the trade-offs, involved in the use of these codes. To illustrate this point, CDUCT-LaRC was used to study benchmark hardwall duct problems to quantify errors caused by wave propagation in directions far removed from that defined by the parabolic approximation. Configurations incorporating acoustic treatment were also studied with CDUCT-LaRC and Q3D-FEM. The cases presented show that acoustic treatment diminishes the effects of CDUCT-LaRC phase error as the solutions are attenuated. The results of the Q3D-FEM were very promising and matched the analytic solution very well. Overall, these tests were meant to serve as a step toward the systematic study of errors inherent in the propagation module of CDUCT-LaRC, as well as an initial test of the higher fidelity Q3D-FEM code.
\end{abstract}

\section{Introduction}

The ability to predict fan noise within complex three-dimensional aircraft nacelle geometries is a valuable tool in studying low-noise designs. Recent years have seen the development of aeroacoustic propagation codes using various levels of approximation to obtain such a capability. For instance, the propagation module of CDUCT-LaRC ${ }^{1}$ utilizes a parabolic approximation to the convected Helmholtz equation formulated by Dougherty 213 This approach affords very efficient propagation calculations, thus allowing solutions for complex three-dimensional geometries to be handled with relatively low computational costs. As mentioned by Lan, ${ }^{4}$ this efficiency can come at the expense of reduced fidelity as the actual propagation direction may diverge from the preferred direction of the parabolic approximation. Additionally, loss of fidelity may occur when reflection and/or scattering of acoustic waves become important. Nevertheless, if appropriate care is taken to account for the assumptions of the parabolic approximation, CDUCT-LaRC provides an efficient framework in which to perform fully three-dimensional aeroacoustic computations. As more extensive three-dimensional codes become available, it would seem appropriate to incorporate these into a framework similar to CDUCT-LaRC and use them in a complementary fashion. For example, a high fidelity design could be achieved using the current CDUCT-LaRC propagation module and a quasi-3D methodology (Q3D-FEM) currently under development at NASA Langley and discussed in companion papers 5,6$]$ These codes could serve as two components of a three-tiered approach. Following this paradigm, CDUCT-LaRC could be used to identify preliminary designs. For proper geometries, Q3D-FEM could then be used to improve the fidelity in regions of the design space where CDUCT-LaRC was not appropriate. Finally, highly accurate, fully 3-D methods could then be used to further refine the results. Additionally, all of the codes could leverage the existing capabilities of the CDUCT-LaRC acoustic radiation module to predict radiated sound power. With these ideas in mind, it is reasonable to attempt a systematic error study to identify a "working envelope" within which to use the various approaches. This work attempts such a study for the CDUCT-LaRC code by working through several benchmark problems for which analytic solutions

\footnotetext{
*Research Scientist, Research \& Technology Directorate, Structural Acoustics Branch, d.m.nark@nasa.gov, Senior Member of AIAA

${ }^{\dagger}$ Senior Research Scientist, Research \& Technology Directorate, Computational AeroSciences Branch, willie.r.watson@nasa.gov, Senior Member of AIAA

${ }^{\ddagger}$ Research Scientist, Research \& Technology Directorate, Structural Acoustics Branch, michael.g.jones@ nasa.gov, Senior Member of AIAA
} 
may be obtained. Some assessment of the Q3D-FEM code is also pursued. In its current stage of development, the Q3D-FEM code is valid in constant area ducts for which the acoustic field is separable in one cross-sectional direction. The rectangular duct geometry chosen for this initial study was selected to satisfy this condition and to provide the ability to address the possible loss of accuracy due to wide angle propagation. In addition, the configuration relates to a grazing flow duct development effort at NASA Langley (SDUCT project). In section II. CDUCT-LaRC calculations are presented for the hardwall version of the rectangular geometry. Comparison of the results serve to illustrate the connection between phase error and cut-off ratio (and hence, propagation angle). The inclusion of acoustic treatment on the duct walls may diminish the importance of this error, as acoustic modes tend to interact more with duct walls as cut-off is approached. Therefore, calculations incorporating acoustic treatment over the full length of the top duct wall are presented in section III Again, comparisons with analytic solutions are used to identify the effects of treatment on phase error where possible. As a side note, treatment was considered on the full extent of the top wall to avoid possible complications caused by scattering from the impedance discontinuity. In addition to the CDUCT-LaRC results, Q3D-FEM calculations are also presented to introduce some of the capabilities of the code. Finally, concluding remarks and further areas of interest are offered in section IV

\section{Amplitude and Phase Error: Hardwall Configuration}

The initial hardwall configuration was used in an attempt to isolate the connection between phase error and cutoff ratio (and hence, propagation angle). The parabolic approximation assumes that acoustic waves propagate in a dominant/preferred direction. Within the CDUCT-LaRC code, this direction is assumed to follow axial grid lines. As the true propagation angle diverges from the preferred angle, a loss of accuracy will occur. This typically manifests itself in the form of phase error, although there may also be an associated (considerably smaller) amplitude error. The straight rectangular geometry considered is $0.152 \mathrm{~m}$ (6 in) by $0.381 \mathrm{~m}(15 \mathrm{in})$, with an overall length of $0.813 \mathrm{~m}$ (32 in). Although 2-D or quasi-3D approaches are reasonable for the chosen geometry, all CDUCT-LaRC calculations were performed on the fully 3-D configuration with a grid of dimension $17 \times 33 \times 65$ in the $\mathrm{y}, \mathrm{z}$, and $\mathrm{x}$ directions, respectively. This was deemed appropriate in that efficiency in fully 3-D computations is a main selling point for CDUCT-LaRC and the benchmark problems offered an excellent opportunity for verification. The code was run on a single $3 \mathrm{GHz}$ Intel Xeon CPU and the average wall clock run time per case was approximately three seconds (this includes the overhead of grid and solution file $\mathrm{I} / \mathrm{O}$ ).

\section{A. Plane Waves}

Plane waves were first considered for a frequency range of $500 \mathrm{~Hz}$ to $2500 \mathrm{~Hz}$ (in increments of $500 \mathrm{~Hz}$ ) with uniform mean flow Mach numbers of $\mathrm{M}=0.0,0.3$ and 0.5. These conditions were chosen in an effort to simulate possible conditions encountered in the aforementioned SDUCT project. For illustrative purposes, figure 1 presents a contour plot of the real part of the acoustic pressure for the $2500 \mathrm{~Hz}$ case with $\mathrm{M}=0.0$. A comparison of the analytic solution and CDUCT-LaRC results along the length of the duct at $y=0.114 \mathrm{~m}(4.5 \mathrm{in})$ and $z=0.0 \mathrm{~m}$ ( $x$ runs axially and the coordinate system is right-handed) are presented in figure 2 This comparison is indicative of the full set of plane wave calculations as the computed and analytic results match very well. This is as expected, since the plane waves propagate along the duct axis (i.e. the preferred/axial grid line direction).

\section{B. Higher Order Modes}

Higher order modes were also considered to provide cases in which the propagation angle actually deviated from the duct axis. In attempting to observe the relationship between error and propagation angle (and cut-off ratio), sources ranging from the $(0,1)$ mode through the $(5,5)$ mode were considered This provided a large array of acoustic sources that, when cut-on, propagate at a wide range of angles relative to the duct axis. Additionally, the source frequency was set to be $2500 \mathrm{~Hz}$ with zero mean flow. This appeared to be a reasonable set of initial source conditions, although further results were obtained for increased mean flow Mach numbers. The analytic solution in this case is provided by the equation describing a right-traveling (positive $\mathrm{x}$-direction) hardwall rectangular duct mode

$$
p_{m, n}^{\prime}(x, y, z)=A_{m, n} \cos \left(\frac{m \pi y}{h}\right) \cos \left(\frac{n \pi z}{w}\right) e^{-i k_{x, m n} x} .
$$

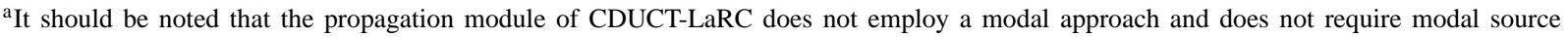
information (although that is generally the case). 
Here, $A_{m, n}$ is the modal amplitude, $k_{x}$ is the axial wave number, $h$ is the duct height, $w$ is the duct width, and $m, n$ are the mode numbers in the vertical $(y)$ and spanwise $(z)$ directions, respectively. To illustrate cases of lower and higher propagation angles (relative to the duct axis), results for the $(0,1)$ and $(2,0)$ modes are presented in figures 3 and 4 . respectively. In the $(0,1)$ mode case, the mode is well cut-on (propagation angle of approximately $10^{\circ}$, cut-off ratio of 5.6) and the numerical result matches the analytic solution very well. Conversely, the propagation angle for the $(2,0)$ mode is approximately $63^{\circ}$ (cut-off ratio of 1.12). As seen in figure 4 , significant phase error is present in the numerical solution. The CDUCT-LaRC propagation module also includes a wide angle correction for the parabolic approximation formulated by Dougherty. Use of this correction improves results for modes closer to cut-off at the expense of increased computation time. In these computations, the wide angle approximation increased computation time by approximately a factor 10 . However, this was caused by the use of a conservative step-size in the ODE solver and could likely be reduced. The wide angle result is also included in the figures (labeled with a "wa" extension) to illustrate the improvement possible with this correction.

In an attempt to quantify the effects of propagation angle on phase error and concisely present comparisons, attempts were made to develop an error measure applicable to the full volume. Initial efforts did not result in a robust formulation possessing the desired behavior. As a result of these difficulties, further comparisons between CDUCTLaRC results and the analytic solution are provided in figures 5 and 6 . The propagation angles for these cases are $27^{\circ}$, $29^{\circ}, 35^{\circ}$, and $44^{\circ}$ (cut-off ratios of $2.24,2.08,1.75$, and 1.43 , respectively) and the results clearly indicate the increase in phase error as propagation angle is increased. It is also interesting to note that very little amplitude error is present, further illustrating the dominance of the phase error. As a side note, the number of plots required to fully examine all comparisons provides some motivation to continue pursuing a general approach to quantifying specific errors.

\section{Effects of Treatment}

As mentioned previously, the inclusion of acoustic treatment on the duct walls may diminish the importance of the errors caused by the deviation of the propagation direction from that assumed by the parabolic approximation. Acoustic waves propagating at larger angles to the duct axis (i.e. closer to cut-off) tend to interact more with duct walls and therefore offer greater opportunity for attenuation. In light of this, the aforementioned rectangular geometry was considered to incorporate treatment of uniform impedance $(\zeta=1.0-0.5 i)$ along the entire length of its upper wall $(y=0.152 \mathrm{~m}(6 \mathrm{in}))$. This allowed the effects of treatment to be isolated while avoiding the further issues of scattering from impedance discontinuities. The analytic solution for this treated case is provided by the equation describing a right-traveling treated duct mode. For a constant impedance on the upper wall, hardwall lower wall, and zero mean flow, this may be written as

$$
p_{m, n}^{\prime}(x, y, z)=A_{m, n} \cos \left(\lambda_{m, n} y\right) \cos \left(\frac{n \pi z}{w}\right) e^{-i k_{x, m n} x} .
$$

Here, the nonzero values of $\lambda_{m, n}$ satisfy the transcendental equation

$$
F\left(k, h, \zeta, \lambda_{m, n}\right)=0
$$

and the transcendental function, $F\left(k, h, \zeta, \lambda_{m, n}\right)$, is defined as

$$
F\left(k, h, \zeta, \lambda_{m, n}\right)=\frac{i k}{\zeta}-\lambda \tan (\lambda h) .
$$

The eigenvalues, $\lambda_{m, n}$, and the axial wave number, $k_{x, m n}$, are related by

$$
k_{x, m n}=\sqrt{k^{2}-\left[\lambda^{2}+\left(\frac{n \pi z}{w}\right)\right]} .
$$

In addition to CDUCT-LaRC, Q3D-FEM was also used for this configuration. The Q3D-FEM code does not incorporate a parabolic approximation and is expected to provide increased accuracy, although at the expense of increased computational effort. The code was used to perform calculations in the $x-y$ plane, as the acoustic field was in fact separable in the cross-sectional $(z)$ dimension. The finite element discretization was composed of 17 elements in the vertical $(y)$ direction and 65 elements in the axial $(x)$ direction. The code was run on eight $1.8 \mathrm{GHz}$ Itanium processors and the average wall clock time for each axial plane case was approximately one second. A contour plot of the real part of acoustics pressure for the least attenuated mode is included in figure 7ffor illustrative purposes. The CDUCT-LaRC and Q3D-FEM results are compared with the analytic solution for the six least attenuated duct modes having a span-wise mode number of zero in figures 8 through 10 . These plots bring about two main points. First, it 
appears that the importance of the CDUCT-LaRC phase error is diminished by the inclusion of treatment. As seen in the various cases, the CDUCT-LaRC results may have some initial errors, but these damp out and the computed solution approaches the analytic result. The second main point of interest is the accuracy of the Q3D-FEM for all of the cases presented. Throughout the figures, it can be seen that the Q3D-FEM results match the analytic solution very well. As a result of these initial comparisons, it is apparent that the current and enhanced Q3D-FEM formulations may provide the higher fidelity methods to be used in the design paradigm described previously.

\section{Concluding Remarks}

This work has described the development of a design framework incorporating tools of increasing fidelity. Two components of such a framework, namely CDUCT-LaRC and the Q3D-FEM have been described and used to study various benchmark cases. The possible errors inherent in the CDUCT-LaRC propagation code were addressed. Initial hardwall benchmark cases with plane wave sources were used to illustrate the accuracy of CDUCT-LaRC for waves propagating in the preferred direction. These were followed with cases involving higher order mode sources to show the relationship between phase error and propagation angle. Initial attempts to formalize this relationships through the use of a proposed error measure met with limited success. However, the effort provided an indication of the utility of a robust approach for verifying the fidelity of various tools. The purpose of extending the benchmark problems to include acoustic treatment was two-fold. First, it provided some further evidence that the inclusion of acoustic treatment helps to diminish the phase error issue of CDUCT-LaRC. However, formalization of this conclusion will require more robust error measures. Secondly, the treated duct cases afforded the opportunity to study the capabilities of the Q3D-FEM code. Results compared very well with analytic solutions and provided motivation for further extension. Overall, these tests were meant to serve as a step toward the systematic study of errors inherent in the propagation module of CDUCT-LaRC, as well as an initial test of the higher fidelity Q3D-FEM. As enhanced verification and propagation tools become available, investigations of the effects of reflections from internal geometries, impedance discontinuities, duct terminations should also be pursued. The end result is expected to be the identification of "working envelopes" within which codes of varying strengths and levels of fidelity could be used effectively for low-noise design studies.

\section{References}

\footnotetext{
${ }^{1}$ Nark, D. M., Farassat, F., Pope, D. S., and Vatsa, V., "The Development of the Ducted Fan Noise Propagation and Radiation Code CDUCTLaRC," AIAA Paper 2003-3242, 2003.

${ }^{2}$ Dougherty, R. P., “A Wave-Splitting Technique for Nacelle Acoustic Propagation,” AIAA Paper 97-1652, 1997. 1999.

${ }^{3}$ Dougherty, R. P., "A Parabolic Approximation for Flow Effects on Sound Propagation in Nonuniform, Softwall, Ducts," AIAA Paper 99-1822,

${ }^{4}$ Lan, J. H., “Turbofan Duct Propagation Model,” NASA CR 2001-211245, 2001. 2005.

${ }^{5}$ Watson, W. R., Jones, M. G., and Parrott, T. L., “A Quasi-3D Theory for Impedance Eduction in Uniform Flows,” AIAA Paper 2005-2848,

${ }^{6}$ Jones, M. G., Watson, W. R., and Parrott, T. L., "Benchmark Data for Evaluation of Aeroacoustic Propagation Codes with Grazing Flow," AIAA Paper 2005-2853, 2005.
} 


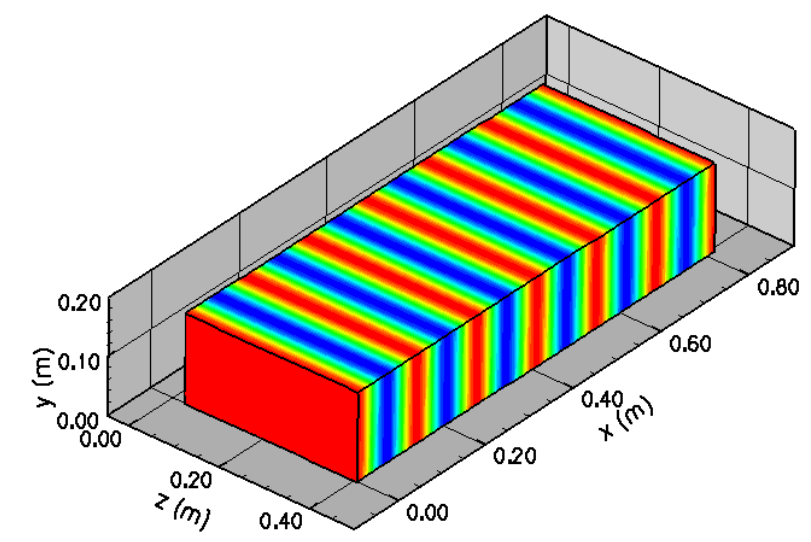

Figure 1. Real Part of Acoustic Pressure [Plane Wave, $f=2500 \mathrm{~Hz}$ ]

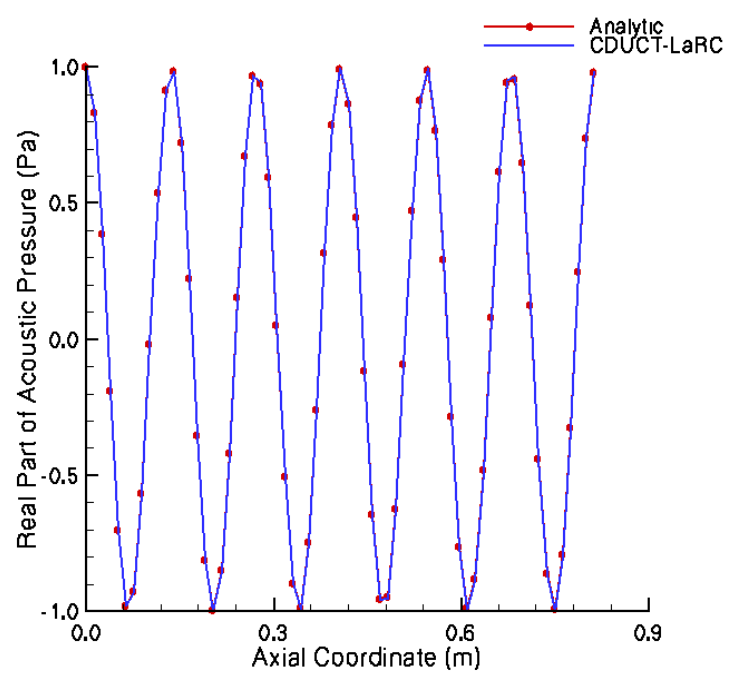

(a) Real Part of Acoustic Pressure

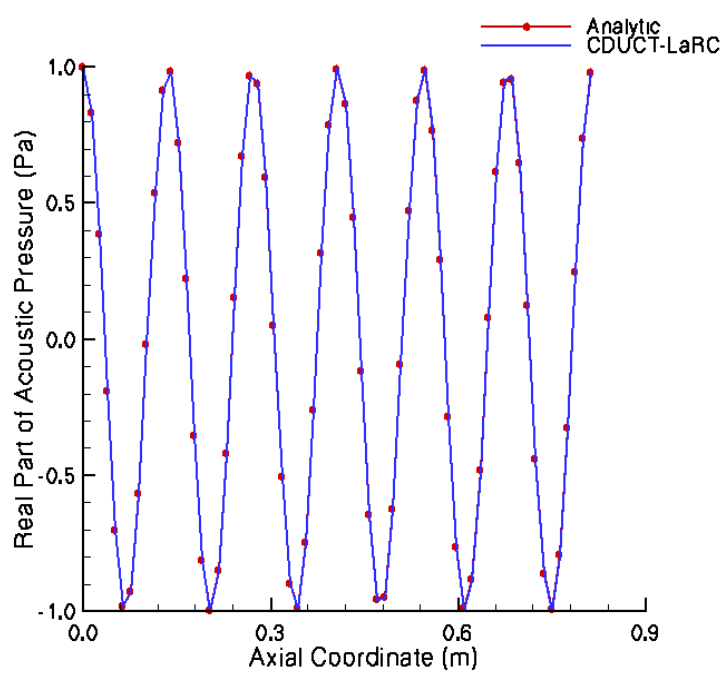

(b) Imaginary Part of Acoustic Pressure

Figure 2. Comparison of analytic and CDUCT-LaRC results [Plane Wave, $f=2500 \mathrm{~Hz}$ ] 


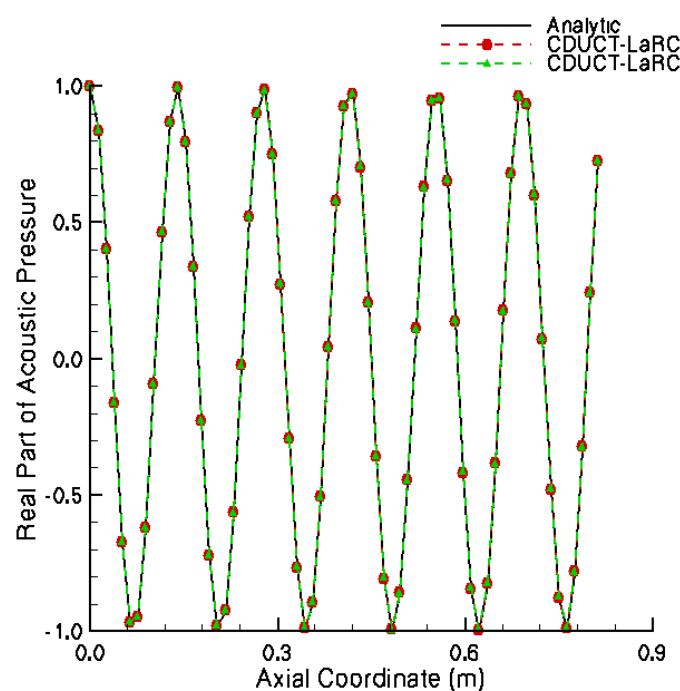

(a) Real Part of Acoustic Pressure

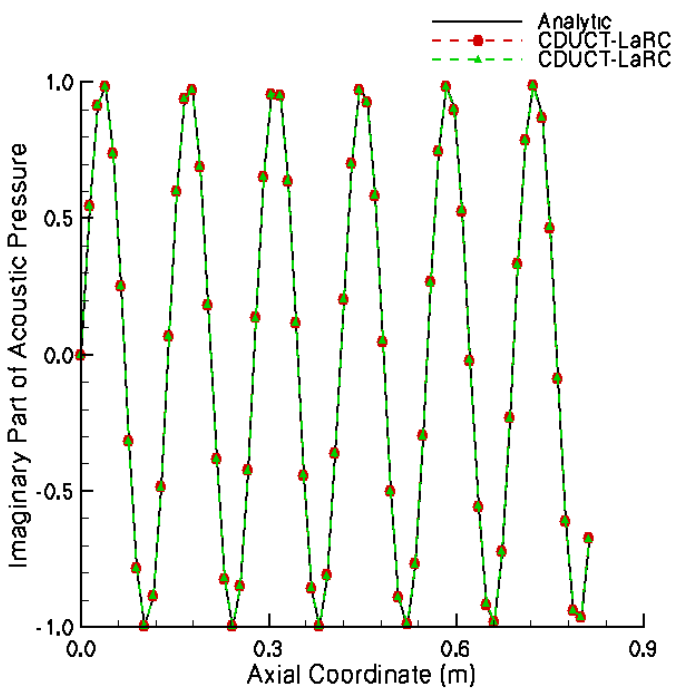

(b) Imaginary Part of Acoustic Pressure

Figure 3. Comparison of Analytic Solution and CDUCT-LaRC Results [(0,1), $f=2500 \mathrm{~Hz}$, Propagation Angle: $\left.10^{\circ}\right]$

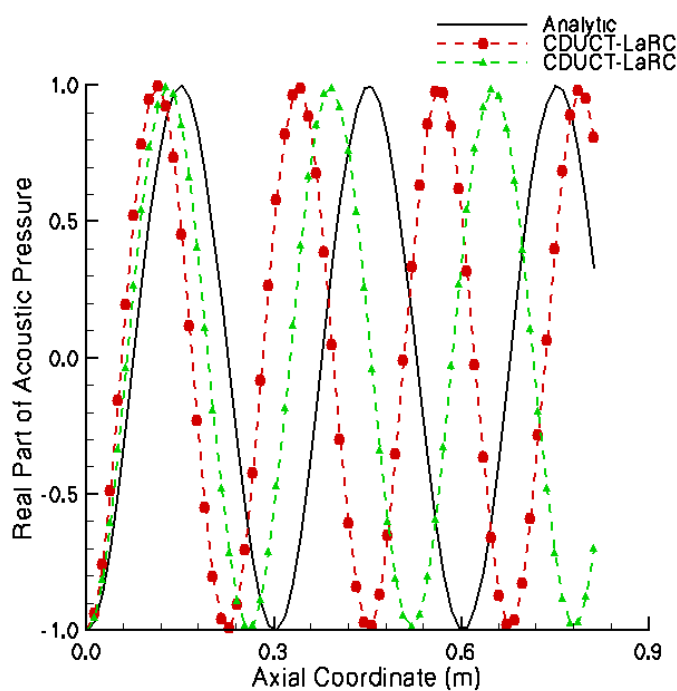

(a) Real Part of Acoustic Pressure

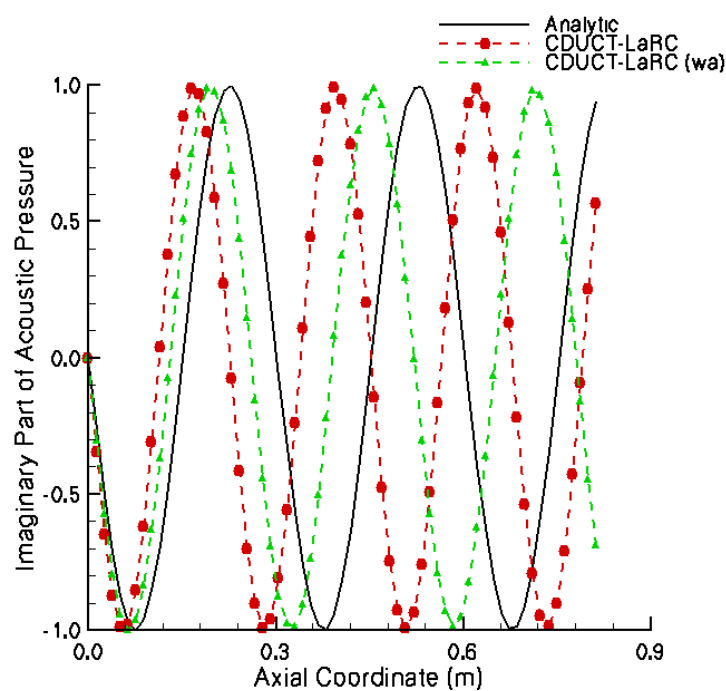

(b) Imaginary Part of Acoustic Pressure

Figure 4. Comparison of Analytic Solution and CDUCT-LaRC Results [(2,0), $f=2500 \mathrm{~Hz}$, Propagation Angle: $\left.63^{\circ}\right]$ 


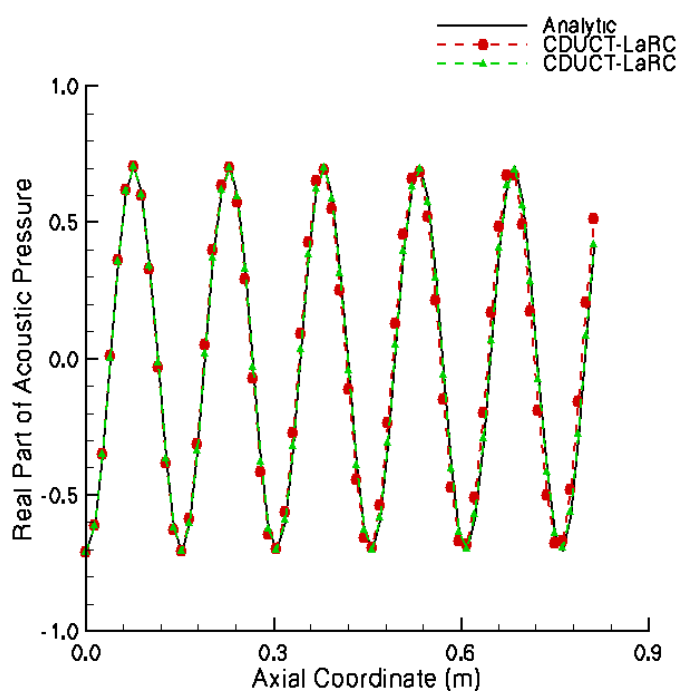

(a) $(1,0)$ Mode, Propation Angle: 27 deg.

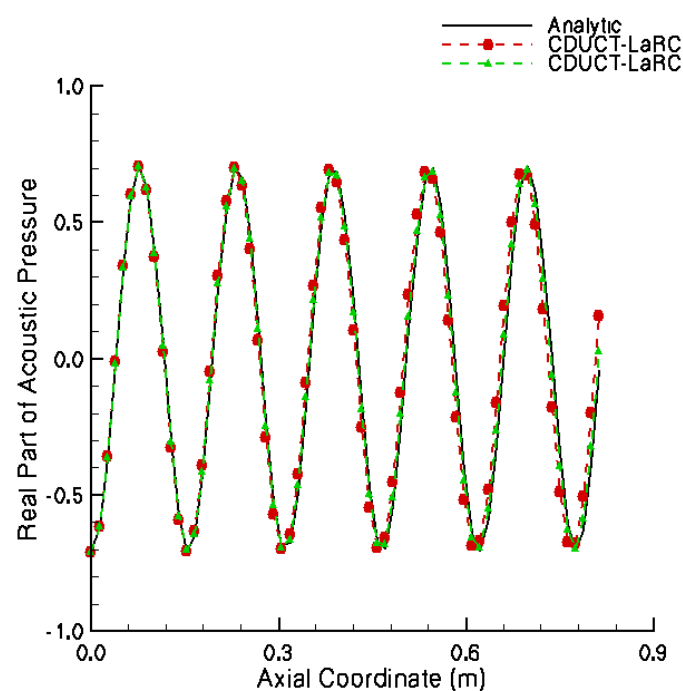

(b) Mode (1,1), Propagation Angle: $29 \mathrm{deg}$

Figure 5. Comparison of Analytic Solution and CDUCT-LaRC Results [ $f=2500 \mathrm{~Hz}$ ]

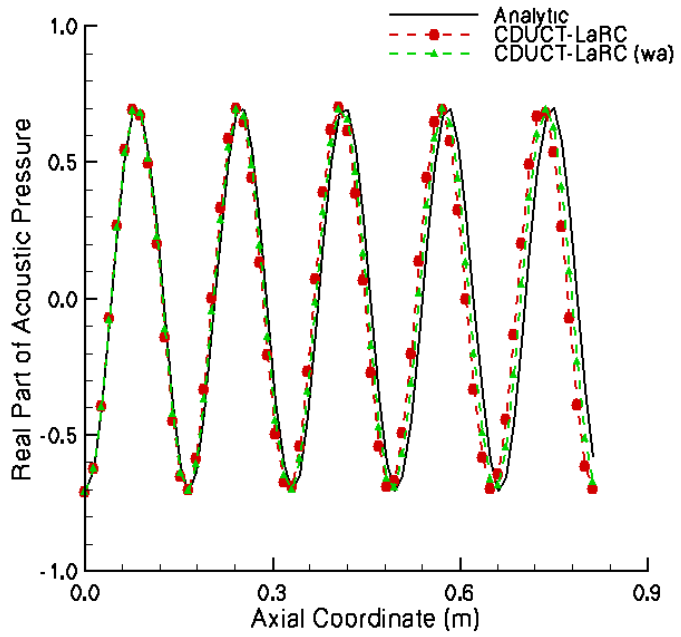

(a) Mode $(1,2)$ Proagation Angle: $35 \mathrm{deg}$

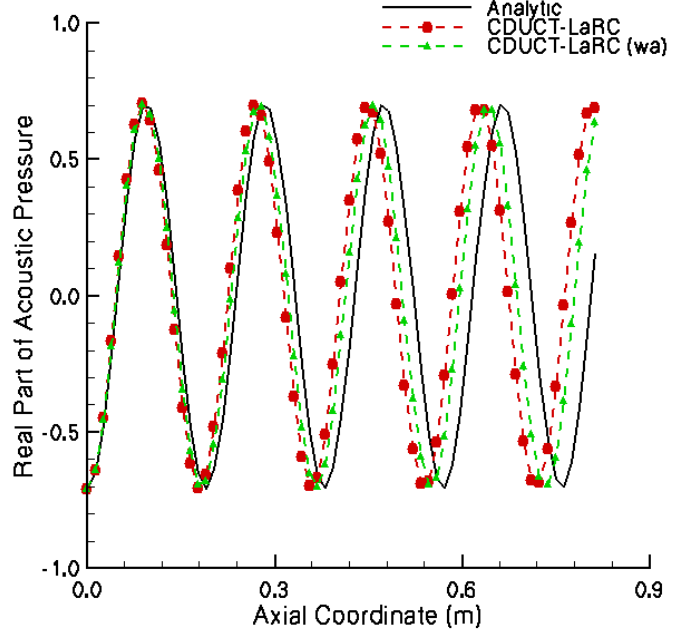

(b) Mode (1,3) Propagation Angle: $44 \mathrm{deg}$

Figure 6. Comparison of Analytic Solution and CDUCT-LaRC Results $[f=2500 \mathrm{~Hz}]$ 


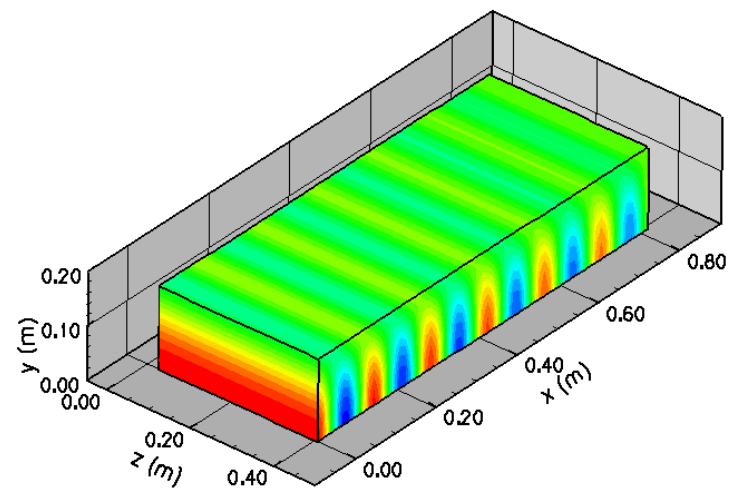

Figure 7. LeastReal Part of Acoustic Pressure [Least Attenuated Mode, $f=2500 \mathbf{H z}, \zeta=(1.0-0.5 i)$ ]

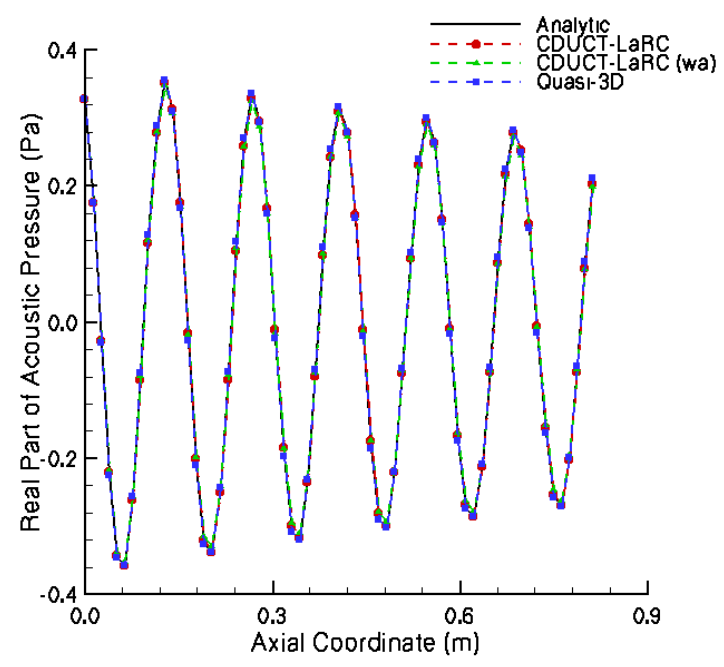

(a) Least Attenuated Mode

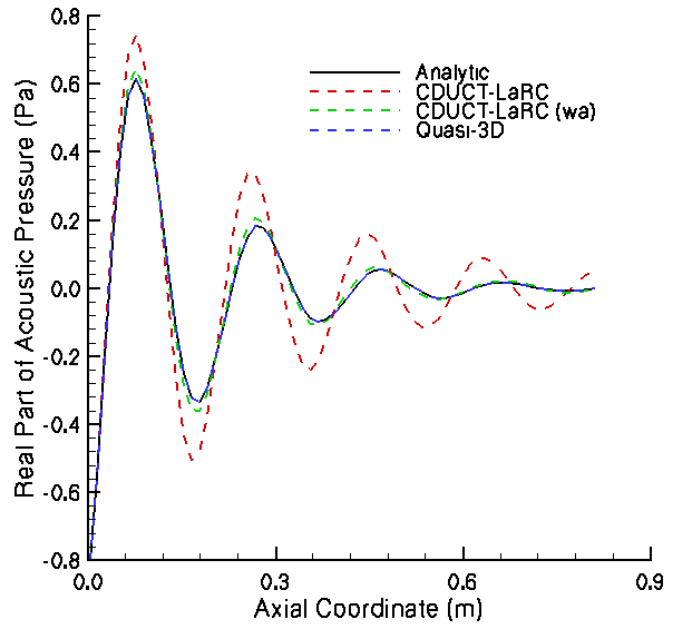

(b) Second Least Attenuated Mode

Figure 8. Comparison of Analytic Solution and CDUCT-LaRC Results $[f=2500 \mathrm{~Hz}, \zeta=(1.0-0.5 i)]$ 


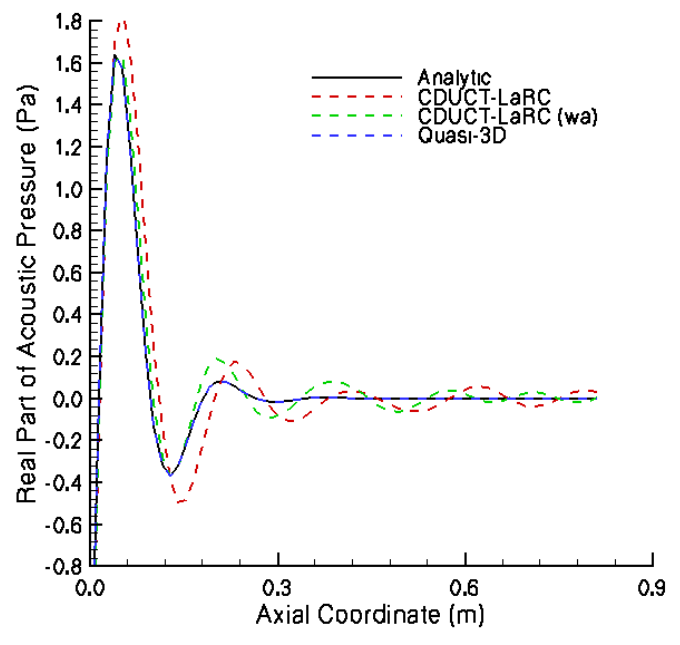

(a) Third Least Attenuated Mode

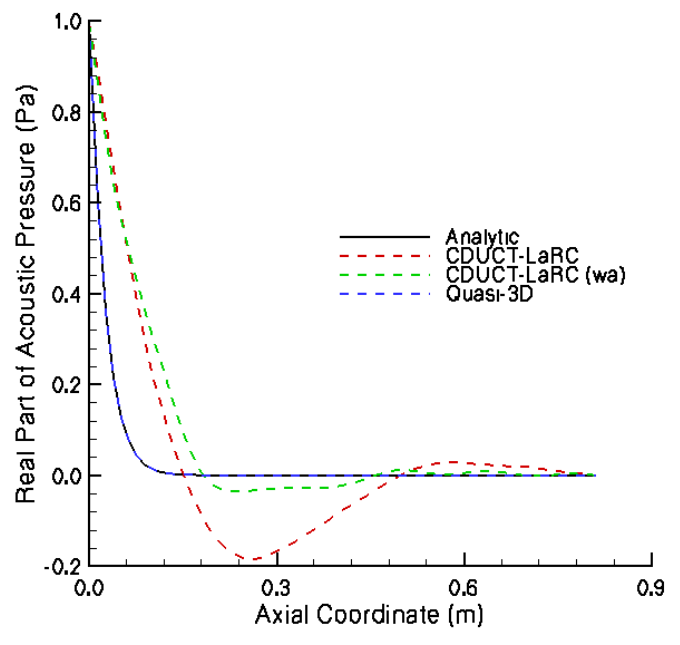

(b) Fourth Least Attenuated Mode

Figure 9. Comparison of Analytic Solution and CDUCT-LaRC Results [ $f=2500 \mathrm{~Hz}, \zeta=(1.0-0.5 i)]$

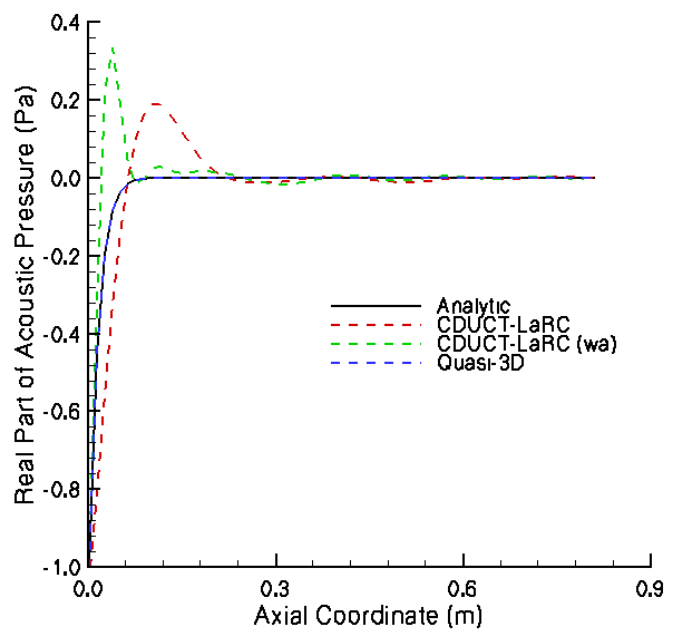

(a) Fifth Least Attenuated Mode

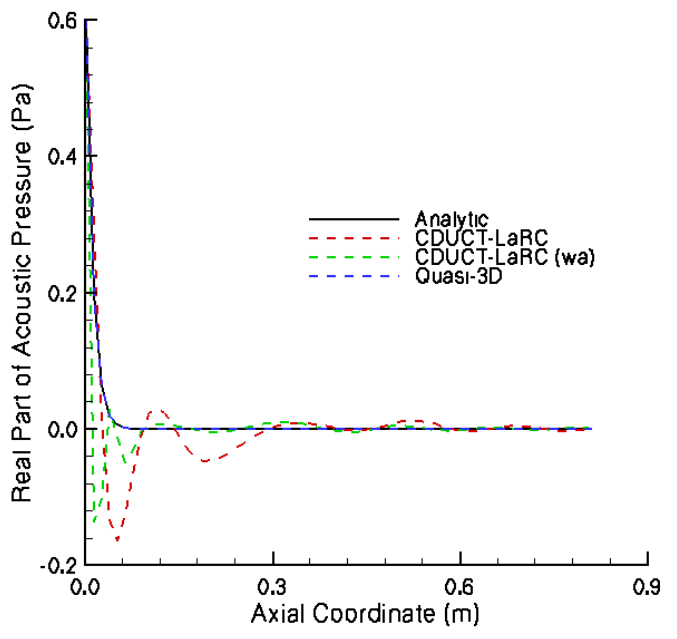

(b) Sixth Least Attenuated Mode

Figure 10. Comparison of Analytic Solution and CDUCT-LaRC Results $[f=2500 \mathrm{~Hz}, \zeta=(1.0-0.5 i)]$

$$
9 \text { of } 9
$$

